\section{Cruce de caminos. Un estado del arte de la investigación-creación}

Gabriel Alba * y Juan Guillermo Buenaventura $^{\text {** }}$

Resumen: Este artículo busca esbozar las distintas acepciones del concepto de "investigación-creación" con el fin de aprovechar su capacidad heurística. Se afirma que sorprende el uso monolítico del neologismo en castellano, en contraste con la variedad de matices de significación que caracterizan al concepto en inglés. Se definen conceptos como "investigación basada en la creación", "investigación dirigida a la creación", "creación como investigación”, el "performance como investigación”, "el uso de las artes en la investigación”, la "artegrafía", la "creación como investigación”. Se realiza una historia del debate sobre el tema y se ponen las bases de un estado del arte guiado por la pregunta: ¿es el arte, investigación? Finalmente, se enfatiza en la creación como investigación en procesos concretos e históricos de creación artística y, a partir de las categorías propuestas por el artista e investigador australiano Graeme Sullivan, se formulan cinco categorías para entender los actos investigativos en los que se involucran artistas y cineastas concretos.

Palabras clave: Investigación - investigación-creación - creación artística - creatividad procesos creativos - cine - prácticas artísticas - arte - actos investigativos en el arte.

[Resúmenes en inglés y portugués en las páginas 48-49]

${ }^{(*)}$ Gabriel Alba es Doctor por la Universidad Autónoma de Barcelona y profesor asociado de la Escuela de Cine y Televisión de la Facultad de Artes de la Universidad Nacional de Colombia. Actualmente es el director del Instituto de Investigaciones Tecnológicas de la Facultad de Artes de la Universidad Nacional de Colombia y líder del grupo Dinámicas Creativas: investigación y creación transmedia de la Escuela de Cine y Televisión de la misma Universidad.

${ }^{(*)}$ Juan Guillermo Buenaventura es Master of Arts de la University of Kansas y profesor asociado de la Escuela de Cine y Televisión de la Facultad de Artes de la Universidad Nacional de Colombia. Actualmente es el Director del Área Curricular de Artes de la Facultad de Artes de la Universidad Nacional de Colombia -sede Bogotá- y miembro del grupo Dinámicas Creativas: investigación y creación transmedia de la Escuela de Cine y Televisión de la misma Universidad. 


\section{Introducción}

No es la primera vez que se intenta construir un estado del arte de la investigación-creación. El más reciente es el de María Victoria Casas (2013), quien hizo un interesante recorrido sobre la manera de entender la investigación en los programas de artes en Colombia y otros países con el objetivo de dar pistas para acreditar los programas de artes de las universidades colombianas. Si bien este estudio muestra un panorama de la discusión, nuestra investigación va por otro lado. No pretendemos circunscribir la investigación-creación al ámbito exclusivamente universitario, sino que buscamos que artistas e investigadores recuerden que sus caminos siempre han estado cruzados y que esos cruces han sido, son y serán de una tremenda fertilidad para el progreso de la existencia humana.

Por eso, el término "investigación-creación" es un neologismo derivado de la composición de dos sustantivos, tal como ocurre con las palabras "bocacalle", "motocarro" o "hispanohablante". Pero más allá de juntar dos palabras, ¿qué ocurre cuando la "investigación" se encuentra con la "creación artística"? Varios elementos surgen de una aproximación a la literatura existente sobre el tema.

Primero. Sorprende que el concepto de investigación-creación se encuentre en los entrecejos de un amplio rango de intereses intelectuales, creativos, académicos e institucionales: desde las llamadas políticas del conocimiento hasta la epistemología del arte y la educación artística, por mencionar sólo tres. En otras palabras, si todavía se tiene la idea, por demás muy extendida, de que la investigación-creación procede exclusiva o predominantemente de las necesidades de legitimación institucional de un saber producto de la creación artística o de la práctica en una universidad, es hora de que esa idea sea cuestionada. La investigación-creación es mucho más que eso.

Segundo. Sorprende también, que el concepto de investigación-creación proceda predominantemente del ámbito anglosajón no-estadunidense: Gran Bretaña, Nueva Zelanda y Australia, aun cuando se haya extendido también a Europa continental y a América Latina. En Estados Unidos y Canadá, el debate ha estado, en buena medida, ausente de la academia o ha asumido una dimensión más "humanista" o "epistemológica", cercana al tema de la educación artística $^{1}$ (Sullivan, 2006, p. 21).

Y es así, porque por más de un siglo la formación de alto nivel de los docentes universitarios de las artes, en Estados Unidos y Canadá, ha sido encomendada a los programas de Master of Fine Arts, o MFA, que en la tradición académica norteamericana, se considera como un título "terminal". Es decir, que habilita para la docencia universitaria sin necesidad de un título de doctorado. Además, el MFA no implica ni la enseñanza ni el desarrollo de competencias de investigación, porque la investigación se concibe como un componente que está implícito en los proyectos creativos. Entonces, los programas de MFA, de dos a tres años de duración, responden al modelo de studio-based learning ("enseñanza con base en estudio"), en la cual el estudiante aprende y/o desarrolla su trabajo artístico bajo la guía de maestros-profesores. En un programa de $M F A$, no existe una "monografía" final, sino que dicho programa termina con la exhibición de una obra artística de alto nivel, en donde cualquier texto diferente a la obra misma, sólo cumple un propósito de archivo académico y/o de explicitación de procesos creativos e investigativos que hicieron posible la obra. Y, si dicho texto es requerido, no forma parte integral y evaluable de la obra (Véa- 
se: https://en.wikipedia.org/wiki/Master_of_Fine_Arts. Recuperado el 16 de noviembre de 2016).

Carentes de esa tradición del MFA, y ante la necesidad institucional de poseer un cuerpo docente con un nivel de formación doctoral, las artes en Gran Bretaña, Nueva Zelanda y Australia, debieron abrirse camino en una muy antigua y arraigada tradición de programas de maestría y doctorado, en donde la investigación era un elemento fundamental y constituyente. En este contexto, los doctorados en artes, tuvieron que inventar el concepto de "investigación-creación" para legitimar sus tesis (Sullivan, 2006, p. 21).

Pero hay, finalmente, una tercera sorpresa: el uso monolítico del neologismo "investigación-creación" en lengua castellana frente a la variedad de acepciones y matices de significación en lengua inglesa. Algunas acepciones, con las que la academia anglosajona ha designado la investigación-creación son: Practice-based research; Practice-led research; Practice as research -PAR-; Practice as Research in Performance -PARIP-; Arts-based research; Arts-informed research; A/r/tography; Research acts in art practice (Sullivan, 2006, p. 20). En este artículo, haremos una mirada panorámica de estas acepciones pero, sobre todo, enfatizaremos nuestra reflexión en el concepto de Research acts in art practice (actos de investigación en la práctica artística).

\section{El horizonte conceptual de la investigación-creación en el ámbito anglosajón}

Los matices lingüísticos en inglés de la relación entre investigación y creación, nos llevan a detenernos en cada uno para comprender sus semejanzas y diferencias.

\section{Practice-based research}

La académica australiana Linda Candy define este concepto como

Una investigación original llevada a cabo para adquirir nuevo conocimiento, parcialmente a través de la práctica y de los resultados de esa práctica. En una tesis doctoral, dicha pretensión de originalidad puede ser demostrada a través de resultados creativos como: diseños, música, medios digitales, performances y exhibiciones. Así, mientras el significado y el contexto de estas pretensiones (de originalidad y de contribución) se pueden describir en palabras, una comprensión completa sólo se puede obtener en referencia directa a dichos resultados (Candy, 2006, p. 1).

Podríamos denominar en castellano este concepto como la práctica basada en la investigación o la creación basada en la investigación. 


\section{Practice-led research}

Linda Candy afirma que este tipo de investigación

(...) se preocupa por la naturaleza de una práctica y lleva a un conocimiento nuevo que tiene significancia operacional para esa práctica. En una tesis doctoral, los resultados del practice-led research pueden ser descritos plenamente en un texto escrito, sin la inclusión de un trabajo creativo. El foco principal de este tipo de investigación es avanzar el conocimiento acerca de la práctica o el avanzar el conocimiento dentro de la práctica. Este tipo de investigación incluye la práctica como una parte integral de su método $\mathrm{y}$, muchas veces, se clasifica dentro del área general de la investigación-acción (action research) (Candy, 2006, p. 1).

Podríamos denominar en castellano este concepto como la investigación sobre la práctica o la investigación sobre la creación o las dinámicas y prácticas creativas.

No sorprende que los conceptos anteriores, la creación basada en la investigación o la investigación sobre la creación, estén imbuidos de un tono institucional ("universidad", "tesis doctoral", etc.). Al fin y al cabo, ambos conceptos proceden de la universidad y de las entidades encargadas de legitimar el saber socialmente constituido, como son los organismos estatales reguladores de la ciencia, la tecnología, la innovación y la educación superior (Sullivan, 2006, pp. 26-27).

\section{Practice as research -PAR-}

Cercanamente relacionado con los anteriores, se encuentra la práctica como investigación, al punto que se podrían utilizar como sinónimos de las dos anteriores. Una definición muy general, pero aceptable, de Practice as research-PAR-es:

La práctica como investigación es una forma de investigación académica que incorpora un elemento de práctica en la metodología o en los resultados de la investigación. En vez de observar la relación entre teoría y práctica como una dicotomía, existe un creciente número de académicos, en distintas disciplinas, que usan la práctica como parte de su investigación (Véase: https://en.wiki pedia.org/wiki/Practice_research. Recuperado el 14 de octubre de 2016).

Podríamos denominar en castellano este concepto como la práctica como investigación o la creación como investigación.

\section{Practice as Research in Performance -PARIP-}

Esta noción ha sido desarrollada por el historiador del teatro británico, Baz Kershaw, y su equipo de trabajo, como parte de la investigación (2001-2005) que dirigió en la Universidad de Bristol, contratada por el Arts and Humanities Research Board-AHRB-, entidad 
estatal británica encargada de la financiación de las artes y las humanidades. El objetivo de esta investigación era indagar por algunas cuestiones creativas y académicas planteadas por la práctica como investigación en el campo de las artes escénicas y/o del espectáculo (teatro, danza, cine, vídeo y televisión) para la Gran Bretaña (Véase: http://www.bris. ac.uk/parip/ . Recuperado el 14 de octubre de 2016). Como un desarrollo de este concepto, se encuentra la noción cercana del Performance as Research-PAR-, que posee su propio grupo de trabajo dentro de la International Federation of Theatre Research-IFTR- (Véase: https://www.iftr.org/working-groups/performance-as-research. Recuperado el 14 de octubre de 2016). Podríamos denominar y sintetizar, en castellano, estos conceptos como el performance como investigación.

\section{Arts-based research}

Desarrollado por los académicos americanos Tom Barone y Elliot W. Eisner, el concepto busca usar las artes como método de investigación para movilizar procesos de educación, desarrollo académico y crecimiento comunitario. Elizabeth Andrews ha definido el concepto como el uso sistemático de procesos y expresiones artísticas (en diferentes formas y disciplinas) como el medio primario para entender y examinar la experiencia de un investigador y las personas involucrados en sus investigaciones. En un sentido amplio, el Arts-based research busca desarrollar, pero también entender, el papel de las artes dentro de las ciencias sociales (Andrews, 2006, pp. 21-22). Podríamos denominar en castellano este concepto como el uso de las artes en la investigación.

\section{Arts-informed research}

El concepto, desarrollado por Gary Knowles y Andra Cole, expertos en la metodología de "historia de vida", está relacionado con el de Arts-based research. El concepto más cercano, dentro de las ciencias sociales latinoamericanas, sería el de Investigación-Acción Participación -IAP-, en la medida en que la investigación busca transformar al investigado y al investigador, e intervenir, definir y proceder desde entornos comunitarios, sociales, políticos e ideológicos. Afirman Knowles y Cole que, “...como un marco para la indagación, el artsinformed research es lo suficientemente fluido y flexible para servir tanto como una herramienta de aprovechamiento metodológico a otros acercamientos investigativos, como una metodología cualitativa con su propio valor" (Knowles y Cole, 2014, p. 59). Podríamos denominar en castellano estos dos conceptos como el arte en la investigación, el arte para la investigación o, en una versión más radical, como la investigación-arte participativa.

\section{A/r/tography}

La práctica de la "A/r/tegrafía", como afirma su más conocida proponente, la profesora canadiense Rita L. Irwin, experta en el campo de la educación artística, es,

to inquire in the world through an ongoing process of art making in any art form and writing not separate or illustrative of each other but interconnected and woven through each other to create additional and/or enhanced meanings. 
$\mathrm{A} / \mathrm{r} /$ tographical work are often rendered through the methodological concepts of contiguity, living inquiry, openings, metaphor/metonymy, reverberations and excess which are enacted and presented/performed when a relational aesthetic inquiry condition is envisioned as embodied understandings and exchanges between art and text, and between and among the broadly conceived identities of artist/researcher/teacher. A/r/tography is inherently about self as artist/researcher/teacher yet it is also social when groups or communities of $\mathrm{a} / \mathrm{r} /$ tographers come together to engage in shared inquiries, act as critical friends, articulate an evolution of research questions, and present their collective evocative/provocative works to others (Véase: http://artography.edcp. educ.ubc.ca/ Recuperado el 14 de octubre de 2016).

En otras palabras, la vida como un permanente "estado de creación" y la creación artística como una forma permanente de investigación. Así, la A/r/tegrafía sería casi una forma vital de acercarse al arte, a la ciencia y a uno mismo, y a su "mundo" social y natural. Podríamos denominar en castellano este concepto como la investigación-creación como forma de vida o, simplemente, como artegrafía.

\section{Research acts in art practice}

Finalmente, está el concepto, medular para este artículo, de la investigación en la creación. La noción del research acts in art practice procede de una idea fundamental: sólo podremos entender, en su justa proporción, la investigación-creación, si comprendemos y estudiamos primero la dimensión real de la investigación que se realiza en procesos concretos e históricamente ubicados de creación artística, sean estos universitarios o no, académicos o no. La idea es que, de alguna manera, todo artista investiga y hay actos investigativos en toda práctica artística y que, por tanto, los actos investigativos son constitutivos y dimensionales de las dinámicas creativas.

La ventaja del concepto de research acts in art practice consiste en que se conecta con la muy antigua tradición de la investigación de la creación y de las prácticas artísticas, representada por la reflexión de disciplinas como la historia del arte, la arquitectura y el diseño; la historia de la literatura; los estudios literarios; la filosofía o teoría del arte y de la imagen; y los análisis de las prácticas de la creación artística. ¿Cómo investiga la creación artística o el diseño? ¿Cómo ha investigado siempre la creación artística o el diseño? ¿Qué operaciones cotidianas de investigación se producen en la creación y el diseño? Preguntas como éstas serían formuladas desde el concepto del research acts in art practice. La bibliografía existente sobre los procesos y dinámicas creativas es muy amplia para todas las disciplinas artísticas involucradas y rebasa las pretensiones del presente artículo. Sin embargo, partiremos del trabajo del escultor urbano, el profesor australiano Graeme Sullivan, y las categorías que ha desarrollado para entender al arte como una práctica investigativa o que conlleva, intrínsecamente, prácticas investigativas (Sullivan, 2006, p. 20). 


\section{Historia del debate}

¿Cuál es la genealogía del concepto de investigación-creación, al menos, en el ámbito académico anglosajón? Más que un debate, lo que se ha producido es una "tensión" intelectual generada por un posible encuentro entre investigación y creación. Esa tensión se produjo cuando la actividad creativa, propia de las artes, el diseño y la arquitectura, se ubicó dentro de la universidad y, en general, dentro de instituciones de educación superior. De esta forma, las primeras escuelas británicas de arquitectura y diseño se crearon en 1840. Pero hay que hacer algunas precisiones. Primero, la enseñanza de las artes, el diseño y la arquitectura se basaron en la noción del studio-based learning, ya definida. Por ponerlo en otros términos: el eje fundamental de las escuelas de artes, diseño y arquitectura no fue necesariamente la investigación sino la preparación de los estudiantes para la vida profesional. Segundo, la investigación universitaria, realizada a lo largo de los siglos XIX y $\mathrm{XX}$ fue, en buena parte, una investigación sobre las artes, el diseño y la arquitectura en disciplinas ya muy consolidadas como la historia del arte, por ejemplo. Y aclarémoslo de una vez: la investigación sobre las artes no es, necesariamente, investigación-creación. Tercero, buena parte de la enseñanza de las artes, el diseño y la arquitectura en Inglaterra se dio en el contexto de los llamados institutos "politécnicos", que nunca han poseído pretensión investigativa y que, ni siquiera, podían ofrecer diplomas de posgrado (Rust, Mottram y Till, 2007, pp. 14-16). Como consecuencia, la investigación de las artes en el sistema de la educación superior a lo largo de los siglos XIX y XX, o fue muy fragmentaria y marginal o no trajo mayor impacto sobre la enseñanza y la formación de profesionales y, en todo caso, no era investigación-creación, tal como la conocemos hoy en día.

Hacia el final del periodo histórico del que estamos hablando, 1840-1992, se produjeron varios procesos sociales y académicos que jalonaron una transformación radical en el estado de las cosas, y pusieron a la investigación-creación en el centro de la escena académica británica. Por un lado, creció la oferta durante la segunda posguerra de programas de doctorado en artes, diseño y arquitectura en Gran Bretaña. Así, entre 1957 y 1975, se otorgaron 38 diplomas de doctorado, en su mayoría en arquitectura, pero también algunos en artes. Como analizan Chris Rust, Judith Mottram y Jeremy Till, no hay evidencia alguna que permita suponer que estas disertaciones doctorales estuvieran trabajando la noción de investigación-creación. En otras palabras, esas investigaciones correspondían al paradigma clásico de la investigación sobre las artes y la arquitectura. Igualmente, entre 1976 y 1985, se otorgaron 100 diplomas de doctorado (44 en arquitectura, 21 en artes y 35 en diseño) que tampoco correspondían al nuevo paradigma.

Puesto que la oportunidad de incorporar la práctica creativa en las disertaciones doctorales ya era una posibilidad establecida por los entes reguladores de la educación de posgrado en artes y diseño a comienzos de los ochenta, en dicha década se produjeron cambios fundamentales en las perspectivas y temáticas de los trabajos doctorales. Entre 1985 y 1995, se otorgaron 40 diplomas de doctorado, de los cuales 12 incorporaron ya alguno de los modelos de la investigación-creación y 28 que, por el contrario, fueron investigaciones clásicas sobre las artes: históricas, antropológicas o educativas (Rust, Mottram y Till, 2007, p. 20). Adicional a lo anterior, se transformaron las realidades industriales, profesionales y sociales en Gran Bretaña. Así, los procesos reales de diseño industrial y arquitectónico requirie- 
ron de una masa crítica de información y datos que sólo podía ser respaldada por procesos sistemáticos de investigación. De esta forma, la investigación aplicada al diseño comenzó, en Inglaterra, a principios de los 60, con el proyecto pionero, liderado por Bruce Archer, acerca de las especificaciones técnicas para el diseño de las camas hospitalarias británicas, y con el establecimiento en 1966, de la Design Research Society, agremiación académica líder en la aplicación de la investigación al diseño industrial.

En el campo de la arquitectura, la Conferencia Oxford de 1958 puso en acción la agenda por un acercamiento investigativo, seguido del hecho de que el Martin Centre, de la Universidad de Cambridge, fue pionero en impulsar una mirada que combinaba métodos analíticos con propuestas de diseño arquitectónico de excelencia (Rust, Mottram y Till, 2007, p. 20).

Nigan Bayasit ha señalado que el desarrollo de productos bélicos (durante la Segunda Guerra Mundial), la carrera espacial, el desarrollo de la teoría de sistemas y de los modelos cibernéticos, hicieron que la academia se enfocara por primera vez en los "métodos del diseño" y en las dinámicas mismas del diseño. En cualquier caso, “...durante los 60, se hizo evidente que los diseñadores no podrían basarse sólo en su habilidad para enfocarse en el producto como centro del diseño".

Design methods people were looking at rational methods of incorporating scientific techniques and knowledge into the design process to make rational decisions to adapt to the prevailing values, something that was not easy to achieve. They were attempting to work out the rational criteria of decision making, and trying to optimize the decisions (Bayazit, 2004, pp. 17-19 y 22-23).

En las artes, se produjeron procesos similares. El monopolio académico que había poseído la historia del arte y la crítica especializada sobre la indagación del arte, empezó, lenta pero inexorablemente, a resquebrajarse y la mirada sobre "la obra" comenzó a dar paso a la mirada sobre "los procesos" (Rust, Mottram y Till, 2007, pp. 14-16).

Las rígidas fronteras entre disciplinas artísticas empezaron a desdibujarse, tanto en la práctica artística misma como en la crítica especializada. Fue todo un periodo de surgimiento de nuevas vanguardias, y se produjo la llamada "descolonización” del arte y la masificación de la educación artística. En el audiovisual, surgieron las "nuevas vanguardias", y empezó el proceso de consolidación de nuevos clásicos como la Nueva Ola Francesa, el Cinemaverité, el Nuevo Cine Latinoamericano, entre tantos otros movimientos de renovación. En el contexto del cine norteamericano, la caída del "sistema de estudio" hollywoodense se había también iniciado. Los sesenta fueron un periodo, además, en que se consolidaron los públicos masivos de la televisión, hambrientos de otras estéticas y otras sensibilidades. Es lo que el profesor colombiano Jesús Martín-Barbero ha llamado el amalgamiento entre cultura popular, cultura masiva y cultura culta. Creemos, a manera de hipótesis, que el surgimiento de la investigación-creación en las artes, como posibilidad, es un elemento adicional que caracteriza esa renovación de época. 
Resumiendo, la investigación-creación sólo empieza a surgir tímidamente en la segunda mitad del siglo XX y, como anotan Rust, Mottram y Till (2007, pp. 16-17), ni siquiera fue una actividad mayoritaria entre profesores.

Hasta 1992, la mayoría no le daba mucha importancia a la investigación y, aún hoy, el concepto de que la investigación avanzada puede contribuir al conocimiento de una disciplina no es ampliamente comprendida, especialmente por parte de profesionales para quienes la "investigación" equivale a una simple recolección de información que fortalece una práctica.

¿Qué estaba pasando en el campo audiovisual? Según Desmond Bell (2006, p. 87), un elemento fue el crecimiento, entre 1986 y 2006, de los programas curriculares en cine o de comunicación audiovisual y multimedia, dentro de la educación superior en Gran Bretaña, Europa continental y Estados Unidos, bajo fuertes presiones políticas para legitimar su "relevancia vocacional", y con millonarias inversiones en laboratorios audiovisuales logrando, efectivamente, que las universidades acogieran la mayor concentración de recursos tecnológicos audiovisuales por fuera de los medios masivos tradicionales. De nuevo, fue una vertiginosa expansión tecnológica y curricular basada en la noción pedagógica del "studio-based learning", -el aprender-haciendo bajo la supervisión de un maestro--, por lo que, como anota Bell, ese crecimiento no siempre estuvo acompañado de una rigurosa reflexión conceptual sobre la relación entre dicha creación audiovisual y la producción intelectual (proveniente de los film studies, los cultural studies o de la mass comunication research). En el contexto australiano, Linda Candy (2006, p. 4) traza el origen del debate sobre la investigación-creación con el surgimiento en 1984 de dos doctorados en escrituras creativas en universidades en ese país.

La llegada al gobierno británico del Partido Conservador, durante los años 80 y 90, con su énfasis en la eficiencia y la evaluación académicas, le dio mayor relieve al debate sobre la investigación-creación en las artes. Según Rust, Mottram y Till (2007, p. 17), una coyuntura decisiva fue la transformación en 1992 en Gran Bretaña, de los tradicionales politécnicos en universidades. Lo que, al menos sobre el papel, implicaba que las artes, el diseño y la arquitectura debían fortalecer su perfil investigativo, sin mencionar el hecho de que, con esa transformación institucional, los politécnicos quedaron habilitados para otorgar diplomas de doctorado. El problema se agudizó con el establecimiento en Gran Bretaña, de programas de doctorado en creación artística bajo el supuesto de que dichos posgrados incluyeran componentes neurálgicos de investigación. Así, entre 1996 y el 2005, se escribieron 406 disertaciones de doctorado en el área. De estas, 148 eran de arte, 99 de diseño y 81 de arquitectura. Según Rust, Mottram y Hill (2007, p. 21), el 20\% de esos doctorados eran "investigaciones acerca de los procesos del hacer o que pretendían indagar acerca de prácticas artísticas contemporáneas, incluyendo trabajos descriptivos y aquellos escritos realizados para acompañar obras artísticas". Por lo visto, la investigación estaba ya ubicada con firmeza en la creación, al menos en Gran Bretaña.

Una parte decisiva del proceso fue el establecimiento, en 1992, del Research Assessment Exercise (RAE), como un proceso público de acreditación y calificación de la investigación universitaria bajo el principio de "quien más investigación realiza, más recursos tiene derecho 
a recibir". Según Bell (2006, pp. 87-88), el resultado de su aplicación en las escuelas de artes fue que los académicos estuvieron bajo una presión mayor para identificar las obras de arte, producidas por sus profesores, en términos de "resultados de investigación", sin que esa identificación entre unos y otros se haya realizado con los debidos cuidados epistemológicos. Si nos atenemos a la reflexión propuesta por Bell, tanto a las entidades reguladoras y financiadoras de ciencia y tecnología como a los y académicos de las áreas de arte y de diseño, "les convino", por las razones más pragmáticas posibles -acreditación y financiación-, ignorar las cuestiones epistemológicas más problemáticas, buscando ubicar las actividades de arte y diseño dentro de un "modelo genérico" del desempeño cuantificable de investigación:

And, if the will was there, then it wasn't too hard to find a way of establishing a suitable fit. Bodies of photographic work, films, sculpture, paintings etc. could be treated as research outputs irrespective of the original motives of their authors providing they found a public exhibition forum paralleling the peer-reviewed publication outlets of written work. This was the primary model employed by the RAE to assess individual academics research output, and art institutes were greatly relieved to find they could reinscribe the art work of their lecturing staff within it and qualify for RAE recognition and funding. Universities began to hire professional filmmakers and visual artists with impressive portfolios of exhibited work to strengthen their research profile in the areas of art, design and the media (Bell, 2006, pp. 87-88).

El resultado fue que muchas instituciones universitarias lograron, con gran eficacia, administrar

(...) the new research economy to great effect and money flowed into 'research active' departments. Almost overnight British universities became important funders of arts projects and of the artists undertaking them, rivalling -and in some areas, threatening- to eclipse arts funding from regional and national arts councils. Despite the apparent rigour of the national research exercise, the reviewers did not seek to critically interrogate the knowledge claims made for this 'research activity' in the arts (Bell, 2006, pp. 87-88).

La conciencia de la necesidad de una reflexión epistemológica y conceptual sobre la investigación-creación convivió, tranquilamente, con un acercamiento mucho más pragmático:

An unstable consensus began to emerge within the creative practice research cadre -by which I mean those active in campaigning for access for the creative arts to public research funding and indeed also active in competing for such funding. This held that generic definitions of research, such as those being toted by the AHRC, might be applicable to the creative arts. Many felt they could sign up to the generic definition of research provided by the research councils, namely, research 'as a process of systematic enquiry whereby new knowledge in a field of study is generated (Bell, 2006, p. 89). 
Se supuso, acrítica y automáticamente, que la producción artística universitaria "equivalía" a investigación y que la exhibición de arte ante colegas artistas, o ante críticos especializados, "equivalía" a la lectura crítica y la evaluación por parte de pares (dentro de las disciplinas académicas tradicionales). El asunto se volvió más agudo con el establecimiento, en 1998, del Arts and Humanities Research Board-AHRB-, antecesora del Arts and Humanities Research Council, o AHRC, con lo cual las formas tradicionales de hacer arte en la universidad, se vieron más presionadas a identificar lo que Bell llamó el "valor agregado como investigación”, para así poder acceder a los fondos de la entidad. Como afirma Bell, esta estrategia de presentar el "vino viejo en odres nuevas", con su resistencia a distinguir entre el "objeto de arte" y los "resultados" de una investigación, mostró rápidamente limitaciones decisivas. Intuitivamente, las instituciones universitarias, y quienes redactaban propuestas de "investigación/obras de arte", se dieron cuenta de que si querían fortalecer su carácter académico, debían ponerle una base conceptual más rigurosa a la investigación-creación. Adicionalmente, se produjo la inclusión de asignaturas de metodología de la investigación en los programas de pregrado y posgrado de artes y diseño. Concluye Bell que, "(...) slowly and surely, questions began to be raised about the epistemic character of creative practice research" (Bell, 2006, p. 89).

Surge, entonces, toda una generación de estudiantes de posgrado en Gran Bretaña que, a mediados y finales de los años 90, son pioneros en la nueva metodología de la investigación-creación que involucra la reflexión sistemática sobre los procesos de producción de una obra de arte, la documentación detallada del proceso, la contextualización de los métodos de trabajo y resultados (dentro de un discurso crítico) e, incluso, cierto grado de sistematización de la experiencia aprendida (Bell, 2006, pp. 90-91). En otras palabras, la academia británica había empezado, finalmente, a legitimar la investigación-creación.

Esta legitimación académica y el debate subsiguiente, se ha extendido a España (a partir de 2002), Chile (a partir de 2013), Argentina y México. En la Unión Europea, las entidades comunitarias se han encargado de unificar criterios y procedimientos que los distintos Estados miembro han desarrollado y precisado. En todos estos casos, el debate sobre la investigación-creación ha estado jalonado por las entidades encargadas de la acreditación universitaria (en el área de artes); por las agencias estatales de financiación de la investigación universitaria; o por las facultades de artes mismas. En Colombia, el debate procede de 2008, y viene siendo tratado por entidades como el Ministerio de Cultura, la Asociación Colombiana de Facultades y Programas de Artes -ACOFARTES-, el Departamento Administrativo de Ciencia, Tecnología e Innovación -COLCIENCIAS- y el Consejo Nacional de Acreditación-CNA- (Barriga, 2011, pp. 317-330). Las universidades y las facultades de artes mismas han iniciado, por iniciativa propia, la definición de la investigación-creación, con miras a legitimar y racionalizar tanto la financiación interna como los esquemas de promoción académica de sus profesores-artistas-investigadores.

Como corolario de todo este proceso histórico, se encuentra la experiencia iniciada en 2002, del Doctorado SACRe, impulsado por Paris Sciences et Lettres-PSL-, un consorcio de universidades públicas del área metropolitana de Paris. El acrónimo "SACRe" representa los conceptos en francés, de: Sciences, Arts, Création, Recherche (ciencias, artes, creación, investigación). El doctorado agrupa a siete escuelas profesionales del Estado francés: el Conservatoire national supérieur d'art dramatique (CNSAD), el Conservatoire national su- 
périeur de musique et de danse de Paris (CNSMDP), la École nationale supérieure des Arts Décoratifs (L'École des Arts Déco), la École nationale supérieure des Beaux-Arts (ENSBA), la Ecole normale supérieure (ENS) y la Ecole nationale supérieure des métiers de l'image et du son (La Fémis). Un estudiante del Doctorado SACRe debe trabajar bajo la dirección simultánea de dos tutores: un artista vinculado al medio creativo y un académico vinculado a la investigación. Dentro del Doctorado SACRe, se busca que:

(...) la production d'œuvres est étroitement associée à une démarche problématisée s'appuyant sur des champs scientifiques potentiellement variés: sciences humaines et sociales, sciences exactes et appliquées, esthétique des différents arts. La thèse vise à définir un positionnement singulier à l'égard de la question de recherche, dans le champ artistique comme dans le champ scientifique ou théorique (Véase: http://www.lefigaro.fr/formation/2012/10/16/09006-2012 1016ARTFIG00573-sacre-un-doctorat-inedit-alliant-sciences-et-arts.php Recuperado el 4 de diciembre de 2016. Y http://www.femis.fr/doctorat-sacre. Recuperado el 4 de diciembre de 2016).

Será una experiencia que todos aquellos interesados en la investigación-creación debemos seguir, con deseos de aprender y de evaluar -amanecerá y veremos-. Pero, como ha insistido tanto Desmond Bell, se requiere, por ahora, de un trabajo detallado y cuidadoso de fundamentación conceptual y epistemológica de la investigación-creación. Y esa fundamentación debe superar, al menos en América Latina, los estrechos y agobiantes límites de las reflexiones introductorias que se escriben para los manuales de presentación de proyectos de grado o en los manuales de procedimientos para la evaluación de esos mismos trabajos de grado, en el área de las artes. Se requiere epistemología en serio de la investigación-creación en América Latina.

Para concluir esta mirada histórica, digamos que durante la primera década del siglo XXI, la investigación-creación ha tenido un nuevo "doliente" y empiezan a configurarse las bases del nuevo paradigma. Un grupo altamente creativo de artistas-académicos-educadores empezó a preguntarse por la investigación-creación como posibilidad cognitiva real, no sujeta a los vaivenes y dudas de funcionarios y financiadores. Así, han salido y circulado los libros y artículos de Tom Barone y Elliot Eisner (2002-2008), la investigación sobre las artes del espectáculo, liderada por Baz Kershaw (2002-2009), los textos de Gary Knowles y Ardra Cole (2001-2008), la artegrafía de Rita Irwin (2004-2013) y el trabajo de Graeme Sullivan (2005-2006). La investigación-creación se ha configurado como un campo académico emergente con debates y dignidades propias.

\section{Elementos del debate}

\section{¿Es el arte conocimiento?}

También: ¿es el arte investigación? Volveremos repetidamente a esta pregunta multiforme y multidimensional. Para empezar, digamos que, si se entiende la investigación como pro- 
ducción y circulación de conocimiento socialmente necesario y transformador, la mayoría de los artistas -de cualquier disciplina- responderían entusiastamente que sí: el arte produce un conocimiento sensible, por lo tanto, el arte sería una forma de investigación que no aborda la ciencia.

Buena parte de la sensibilidad colectiva de la humanidad ha sido moldeada y alimentada por el arte. La poesía y la narrativa, pasando por la pintura y el cine, han alimentado nuestros mundos interiores y nos han dado herramientas para la transformación de nuestros símbolos y nuestras relaciones sociales. El arte crea y procesa conocimiento, signicidad e información, que están en la base (y son la materia prima) de la "humanización del ser humano". Visto desde el punto de la vista de un humanismo amplio y generoso, y de una noción igualmente amplia y generosa de investigación, no existen dudas de que el arte es investigación, indagación y construcción de un conocimiento social e históricamente vital, y buena parte del impacto del arte en los seres humanos radica en su carácter performativo: transforma, a veces intangiblemente, a veces con fuerza directa, las vidas de quienes lo consumen y gozan.

Ahora bien, la anterior reflexión no ha podido responder suficientemente a la pregunta ¿es el arte investigación? y es, claramente, insuficiente desde la tradición de las ciencias, tanto sociales como exactas:

(...) the notion that creative practice itself -with its enthusiasms and confusions, expressivity and sheer immanence- could be the crucible for a process of systematic research investigation remains a harder sell within the wider academic community. And yet, that is precisely what has to be established if the specificity of academic research in the creative arts disciplines is to be delineated and given due intellectual recognition (Bell, 2006, p. 85).

\section{¿Cuál es la relación entre arte e investigación?}

Una dimensión de análisis es el uso de las actividades de investigación dentro de la actividad artística. ¿Hay investigación en las artes? Con su énfasis en el cine, Desmond Bell afirma que todos estamos dispuestos a aceptar que las actividades de investigación pueden formar parte, y de hecho lo hacen, del desarrollo de un filme, y de cualquier obra de arte (Ibid, pp. 85-89). Pero de nuevo la duda: ¿puede llamarse "investigación” a esa investigación? ¿Bajo qué condiciones puede esa investigación convertirse en investigación disciplinar e institucionalmente legitimada como tal?

En términos de las ciencias sociales tradicionales, se puede realizar una lectura de la novelística contemporánea. Por ejemplo, la escritura de novelas como La guerra del fin de mundo y El paraíso a la vuelta de la esquina, de Mario Vargas Llosa; o El nombre de la rosa, de Umberto Eco; o El General en su Laberinto, de Gabriel García Márquez, por citar sólo algunas de tantas, exigieron procesos dispendiosos de recolección y sistematización de datos e información. Sin embargo, ¿cuándo, el visitar lugares y hablar con testigos, se vuelve etnografía? ¿Cuándo el recoger, ordenar, leer e interpretar gran cantidad de textos se vuelve análisis de discurso? ¿En qué punto el conversar se troca en entrevista etnográfica? ¿Cuándo la búsqueda de información de archivo se vuelve historia? ¿Cuándo el rastrear percepciones 
académicas se convierte en historiografía? ¿Cuándo el buscar antecedentes pasa a la construcción de un estado del arte? ¿Cuándo una indagación sobre imágenes para dirección de arte o dirección de fotografía se vuelve historia del arte? ¿Son La guerra del fin de mundo, El paraíso a la vuelta de la esquina, El nombre de la rosa y El General en su Laberinto ejemplares de etnografía, análisis de discurso, historia, historiografía, estados del arte e historia del arte? Pero la pregunta es más amplia, ¿ison necesarios la etnografía, el análisis de discurso, la historia, la historiografía, los estados del arte y la historia del arte para la creación? ¿Cuándo la "investigación para la creación” se vuelve simplemente "investigación y creación”? ¿Cuál es la relación entre investigación y creación en la investigación-creación?

Volvamos a nuestra primera reflexión. Según afirma Bell, el problema de aceptar, sin un mínimo filtro crítico, que el trabajo artístico es "investigación”, que se produjo en la academia británica en los años 80 y 90, no fue tanto que alguien dudara que los artistas se involucran en actividades de investigación, sino que esas actividades -investigación de materiales, medios y estrategias creativas- correspondieran a los cánones de la investigación tradicional, en las ciencias sociales, físicas e, incluso, en las humanidades.

Such institutional definitions placed great emphasis on the systematic and replicable character of research methodologies employed and on the formulation of key research questions. The obligation was for artists to articulate their research questions as distinct hypotheses capable of being tested against specifically gathered data. As we have argued, the research activities of artists and media makers undertaken prior to a creative project and integral to its successful realisation, has as its specific focus the development of that project. It is not primarily concerned with arriving at generalisable conclusions about art and design practice per se nor with the nomological concerns that motivate the natural sciences. (...) The focus of such reflection is, as I have suggested, usually pedagogic. It generally involves getting a particular student artist or group of students to understand their work better with reference to public cultural considerations.

In other words, while an artist's research facilitates their practice, the primary focus of their creative process remains the art object -'making work'- and not an abstract knowledge object. However, research councils like the AHRC remain nervous about funding creative practice projects such as films, photography projects, installations, performances, where the 'research value added' component cannot be delineated from the vehicle of the creative practice and evaluated as a separate 'deliverable'. So the pragmatic challenge for the creative arts within the university has been to preserve the character of its distinctive pedagogy while being able to compete effectively within the economic logic of research performativity (Bell, 2006, pp. 89-90).

Estaban quienes defendían (Darren Newbury, por ejemplo) la aplicabilidad plena del paradigma de la investigación en ciencias sociales a la creación en arte y diseño. Si seguimos el panorama delineado por Bell, para estos autores, la investigación-creación sería, más bien, una "creación basada en la investigación” y el modelo ideal de "investigación” sería aquel, 
epistemológica y metodológicamente hablando, propio de disciplinas "duras" en datos e información, como son la sociología, la antropología o la psicología empíricas (Bell, 2006, pp. 91-92).

Newbury sketches here what philosophers of science refer to as the deductive - nomological model. Historically, this was advanced by logical empiricists as an account of the advancement of scientific knowledge in physics. Later it was argued that this model could be applied to the behavioural and social sciences. Scientific discovery, it is held, involves formulating hypotheses capable of being confirmed or refuted by adduced evidence. Ideally, these hypotheses should flow deductively from law-like propositions about the natural or social world. It is assumed that we reformulate these nomothetic statements on the basis of whether the empirical hypotheses which flow from them are confirmed or disconfirmed in experiment. As has been often noted, this model is not a descriptive one even with regard to natural science but is normative in character. Positivism, in other words, 'wants to articulate an ideal of science for a group of disciplines. The group in question includes the natural sciences and the so called behavioural sciences (Radnitzky, 1968, p. xvi).

La propuesta de Bell es clara: ¿Qué modelo de ciencia asumen y defienden quienes pretenden que la creación artística sea equiparable a la ciencia? ¿Qué tan empiristas y positivistas son? ¿De qué tipo de ciencia estamos hablando cuando hablamos de ciencia? ¿De qué tipo de ciencia social estamos hablando cuando hablamos de ciencia social? Insinúa Bell que, de pronto, este acercamiento ("ciencia igual a arte") podría beneficiarse de una mirada más atenta a las disciplinas humanas no empíricas, como la investigación histórica y/o la hermenéutica y, por tanto, alejarse de aquellas miradas científicas basadas en el empirismo lógico. La insinuación de Bell es interesante: la investigación-creación, por ejemplo, de un largometraje de ficción, ¿podría relacionarse con aquellas disciplinas más conexas, como la teoría de la imagen, los film studies, la historia y estética del audiovisual, en vez de referirse a la sociología, la antropología o la psicología? Como cree Bell, el modelo empirista de las ciencias sociales -filosóficamente desacreditado, históricamente desinformado y éticamente cuestionable- y que ha sido objeto de más de cincuenta años de duros cuestionamientos desde las teorías críticas del conocimiento, ahora es aplicado al mundo de la investigación del arte y el diseño. Lo que se sacó por la puerta trasera de las ciencias sociales, se coló subrepticiamente por la ventana de la investigación-creación.

Of course few of those who wish to articulate art and design research methods within a positivist paradigm adhere in any rigorous way to the fallibilist imperative which is central to the logical empiricists epistemological claims. Rather, the pseudo-positivism now present in the research application formulations of some arts practitioners and most traditional arts and humanities scholars, functions primarily as a discourse to legitimize claims for support for art and design projects to bodies like the AHRC and other funding bodies. We have all learned to talk the talk, and the talk is a sub-positivist discourse of 'research 
questions', 'methods of investigation', 'data collection and analysis', 'milestones' and 'deliverables' -a discourse, in other words, of bureaucratic accountability rather than of scientific discovery. As critical theorists have reminded us, positivism was always complicit with instrumental rationality and the 'iron cage' of bureaucratic control. It remains so (Bell, 2006, pp. 91-92).

Finalmente, y relacionada con todas estas preguntas, se encuentra la duda sobre la relación entre investigación-creación y calidad artística. Como muchos directores y tutores de trabajo de grado pudieron observar, se produjeron obras de arte de dudosa calidad artística basadas en investigaciones de gran calidad. ¿Es la investigación un prerrequisito de la calidad artística? ¿Por qué hay casos, no infrecuentes, de diseño y arte de calidad, que no han requerido investigación alguna? Se pregunta Bell: "Si la investigación para la práctica creativa no mejora esa práctica, ¿para qué entonces la investigación?” (Ibided).

\section{Análisis crítico-conceptual y arte: ¿investigación-creación?}

Otro elemento de análisis se encuentra en las actividades conceptuales y analíticas que, en diferentes grados, momentos y modalidades, desarrolla todo artista. En otras palabras, ¿puede llamarse investigación al desarrollo de marcos críticos de análisis y comprensión de la obra de arte? La sugerencia de Desmond Bell (2006, p. 86) a este respecto es clara: ese tipo de investigación, -la producción de marcos de comprensión críticos-forma parte del quehacer creativo mismo. Pero, ¿ forma el quehacer crítico-conceptual parte indispensable de la investigación-creación?

Íntimamente relacionada con la pregunta anterior, está la cuestión de si ¿se puede hacer arte y creación en y desde la universidad? Todos estaríamos dispuestos a aceptar que la universidad es, históricamente, un ambiente institucional propicio para la creación artística. Ahí no está la discusión. De hecho, en áreas como las artes plásticas o la música, algunas de las más importantes obras de arte de los últimos 200 años han sido producto, directo o indirecto, del trabajo al interior de una institución de educación superior o, al menos, de una relación de enseñanza-aprendizaje (Bell, 2006, pp. 85-86). La pregunta por la universidad es, claramente, una pregunta por el carácter "pedagógico" que ha tenido la reflexión -y por derivación- la investigación sobre las artes. Dicha tradición crítica surge en, y desde, procesos de enseñanza/aprendizaje de las mismas artes. Así, argumenta Bell, la enseñanza/aprendizaje de un arte exige, aun cuando sea mínimamente, el ordenamiento de un marco conceptual y crítico:

Of course, it could be argued that arts pedagogy itself involves a critical dimension and that historically this pedagogy, rather than 'pure' research, has been the locus of critical reflection and theorising within the creative arts (one thinks of the pedagogic writings of Malevich, of those of the Bauhaus Masters or Eisenstein's extensive reflections as a teacher on filmic form and meaning). Certainly, as teachers, we are concerned with assisting our students to develop their work and to understand it through its critical contextualisation and interrogation within a public culture (Bell, 2006, pp. 87-88). 
¿Son esos marcos críticos, ahora inmersos en un contexto pedagógico y de enseñanzaaprendizaje, investigación en cuanto tal? Bell afirma que:

One of the weaknesses of many of the media arts programmes which have emerged out of media studies, is that while they have offered students some experience of media production, they have been slow to embrace a projectbased arts pedagogy which gives the formal critique a central role in the development of a student's creative abilities. Indeed, a commitment to make sense of work within a public cultural context and with reference to available modes of critical thinking is present in the best film and imaging programmes where the acquisition of technique and mastery of convention and form is balanced with the teaching of practices of self-criticism and public accountability (Bell, 2006, pp. 87-88).

Marta Lucía Barriga (2011), retomando a Roberto Fajardo, ha explorado la noción del "educador-artista-investigador": un artista que fluye entre la sistematización de su práctica artística, su difusión con vistas al enriquecimiento creativo en la enseñanza-aprendizaje, y la creación misma. De alguna manera, la investigación-creación se encuentra a gusto con la pedagogía y con la educación artística.

Otro elemento de estas diferencias es el tema de la comunicabilidad del conocimiento adquirido, por ejemplo, en el contexto de la investigación-creación doctoral. Desmonnd Bell (2006:90), ha calificado los requerimientos escritos de enunciación y elaboración teórica de nivel doctoral, como una especie de "autoderrota" de aquellos que buscan la autonomía del conocimiento en las artes. La pregunta amplia es, por supuesto, si la obra de arte, para ser obra de arte, tiene que someterse a la discursividad de la investigación tradicional.

\section{La investigación-creación y la creación tradicional}

El debate anterior nos da luces acerca de las diferencias existentes entre la investigacióncreación, por un lado, y la práctica artística tradicional, por el otro. Hay quienes, el documentalista británico Erik Knudsen por ejemplo, defienden la "inabordabilidad" radical de la creación artística. Para estos autores, el arte es una actividad irracional que no admite sistematicidad científica. En otras palabras, ¿es el concepto mismo de investigación-creación, desde su génesis, una imposibilidad y un sin-sentido? Como reacción a los excesos positivistas del otro extremo, ya esbozados,

(...) many creative arts academics reject what they perceive as the scientistic character of the dominant discourse about research. The drift towards a subpositivist discourse in art and design research has led to outbreaks of romantic resistance and the 'return of the muse (Bell, 2006, p. 92).

La posición de estos autores busca defender la creación por sobre la investigación, y la expresión artística como una actividad básicamente irracional e, incluso, mística. Bell toma distancia de este acercamiento. Para él, es difícil ver cómo el uso de tal misticismo, y de tal 
abandono del método crítico, puede ayudar a avanzar nuestras discusiones acerca de la investigación-creación. Concluye que:

Neither of these essentially nineteenth century positions seems to have much to offer in the contemporary task of clarifying the status of the knowledges generated in and through creative practice. Both seem avoidance strategies, a supplementary discourse obscuring our critical understanding of the interaction between the cognitive and aesthetic dimensions of art practice (Bell, 2006, p. 92).

Un elemento que nos da luces sobre la diferencia entre la investigación-creación y la creación tradicional, es un dato de la realidad de la práctica artística misma: muchos académicos tienen su propia práctica creativa por fuera del mundo académico. Cuando están en un contexto institucional universitario, hacen investigación-creación, cuando están por fuera de ese contexto, simplemente son artistas:

This raises the tricky question identified by the PARIP team, 'What makes an instance of practice "count" as research?' (op.cit, p. 118). Is it merely the location of the practice project within the academy that establishes its research status? This is patently not so as teachers of drama in universities often produce work which plays in university theatres and which has no intrinsic research aspiration. Surely practice as research must have different methods and planned outcomes to 'pure', i.e. professional, practice.

The water has been muddled further by RAE assessment practices in the field of drama and the performing arts. These evaluations have treated professional productions and art works attributed to academics and entered for the national research assessment exercise as quantifiable research outputs, irrespective of whether the authors themselves conceived of their arts works in this way. Lecturers in arts colleges have always produced their own art works and exhibited these. Only recently however, and in response to institutional pressures, have they been tempted to describe this activity as research (Bell, 2006, pp. 93-94).

Para radicalizar el debate está el otro tema del carácter "efímero" de muchas de las prácticas del arte y, en especial, de las artes del performance. ¿Cómo registrar un performance en toda su amplitud y complejidad, si sólo podemos acceder a él a través de la inmediatez de su puesta en escena -en el aquí y en el ahora-? En muchas disciplinas artísticas, el "resultado" de un proceso creativo son "obras" materializadas en el espacio y/o el tiempo. ¿Es así en las artes escénicas? ¿Qué implicaciones tiene ese carácter "efímero" para ciertas disciplinas artísticas escénicas que buscan también ubicarse en el paradigma de la investigacióncreación? Sin embargo, estaríamos desconociendo la complejidad epistémica del problema si lo reducimos a un asunto de "registro académico". En realidad, el tema está, de nuevo, relacionado con la centralidad del discurso escrito en el mundo académico tradicional y dentro de las prácticas de circulación del conocimiento en las disciplinas tradicionales. 
PARIP has provided a useful forum for airing a series of issues about the relationship between professional practise per se and its operationalization as a research method where the primary object becomes an epistemic rather than an art one. Its discussants have explored how the methodology of PAR resembles and differs from that operating in other disciplines in the arts, humanities and social sciences. They have posed questions about the relationship between the propositional forms of knowledge and writerly practice characteristic of traditional scholarship, and the tacit and embodied forms of visually-led knowledge found in art practice. Departing from Phelan's concern with the ontological specificity of performance, they have questioned the role of written report and textual analysis characteristic of traditional research documentation and have highlighted the forms of reflexive analysis encoded in the performance work itself.

So far so good. But after these extensive PARIP deliberations, are we any clearer about the epistemic character of practice as research? Far from it being the case that the, 'time for questions is over', as Piccini and Kershaw playfully suggest in their review of the work of PARIP (op. cit, p. 118), we haven't really started asking the right questions. Apart from a brief skirmish with positivist models of knowledge generation (indicted for privileging propositional over experiental knowledge), and a parading of a lineage of phenomenological and post-critical philosophers from Heideigger to Polanyi, held to have shaped performance theory's concern with the embodied subject and with reflexivity, the PARIP researchers seem to have little to say about the conditions of possibility of knowledge through art practice. Yet, the imperative within creative arts research remains to, 'do the epistemological spadework' required to provide answers to these methodological queries, however tentative and provisional these might be (Bell, 2006, p. 94).

\section{La investigación en el arte}

Finalmente, debemos preguntarnos por la investigación-creación en su acepción en lengua inglesa como research acts in art practice; es decir, por la investigación como una dimensión constitutiva del trabajo artístico. Como ha propuesto Graeme Sullivan, a quien seguiremos de cerca en este tema,

When art practice is theorized as research, it is argued that human understanding arises from a process of inquiry that involves creative action and critical reflection. As a significant means of human understanding, art practice is very mindful work as it makes good use of cognitive processes that are distributed throughout the various media, languages, and contexts used to frame the production and interpretation of images. There is an inherently transformative quality to the way we engage in art practice, either as learners or teachers, and this dynamic aspect has to be embraced if the idea that the studio experience can be conceptualized as research is to have legitimacy. The researcher and the researched are both changed by the process because creative and critical 
inquiry is a reflexive process. Similarly, a viewer or reader is changed by an encounter with an art object or a research text as prior knowledge is troubled by new possibilities (Sullivan, 2006).

Sullivan ha propuesto cuatro categorías para entender los actos investigativos en los que se involucre un artista:

- Actos teoréticos.

- Actos formativos.

- Actos interpretativos.

- Actos críticos.

Desde esta investigación, proponemos una quinta categoría:

- Actos metodológicos.

Empecemos a desglosar cada una de estas nociones. El arte no es pura "sensibilidad": el arte implica y/o requiere operaciones conceptuales complejas: son los actos teoréticos. Sullivan enfatiza que el pensamiento siempre ha formado parte del quehacer artístico:

...artists have always been deep thinkers. What has expanded, however, is the range of conceptual tools, creative approaches, and communal contexts, within which artistic practice takes place. A characteristic of this practice shows that artists periodically "think in a medium", "think in a language", and "think in a context" (Sullivan, 2004). The creative and critical intent of artists who "think in a medium" emphasize formal and expressive properties that are revealed by their explorations of media. For artists who "think in a language" a rich interpretive landscape is opened up because art experience is extended through dialogue and discourse. On the other hand, artists who "think in a context" are interested in creating critical artistic encounters that change the way we think about things around us. Consequently, in considering art practice as theory, the way artists think about studio processes as forms, ideas and actions (...), gives a sense of the rich theoretical underbelly that propels art inquiry. Conceiving art practice as a theoretical act within a framework of inquiry sets in place the prospect of doing research in artmaking. When used as a site for research, art practice brings into play the seamless relationship between the 'researcher' (artist) and the 'researched' (art practice) and this builds on all the discursive arguments that disrupt untenable dichotomies such as the fictive subjective-objective divide. Or to put it another way, the task is to claim a legitimate place for the artist in the research process, and to do this, other research acts can be taken up (Sullivan, 2006, p. 30).

Desmond Bell estaría de acuerdo. Según este autor, el desarrollo de cierto nivel crítico como crítica conceptual- forma parte de un quehacer artístico complejo. Así, siguiendo 
a Kathyrine Grushta, Bell (206, pp. 86-87) sostiene que existe un "ciclo hermenéutico" de involucramiento y alejamiento afectivo, del hacer y del criticar.

Sullivan (2006, p. 31) también ha propuesto que un artista desarrolla actos formativos. ¿Qué son los actos formativos?

Although the mind is the medium that most clearly shapes art practice, for many art researchers art materials are still the most tangible means that give form to imaginative thought. Therefore, when under taking studio research where there is a focus on structural qualities among other interests, the artist really does think in a medium. In this process the artwork becomes the primary site and source of knowledge, just as with painting where questions, problems, and insights emerge as part of the practice. Research acts such as visual problem finding and problem solving are characteristic of this kind of inquiry process whereby forms, materials, properties, and qualities become the means by which concerns are explored and expressed. This reflective intent fuels an exploratory tendency as new forms and images are created, and these open up the possibility of new meanings.

De acuerdo con Sullivan, una característica de estos actos formativos es que la comprensión emerge dentro de un 'proceso de experimentación con medios y que ese conocimiento performativo puede equipararse a estrategias tradicionales como la observación y la confirmación empírica.

Sullivan también ha propuesto que un artista desarrolla actos interpretativos:

For those art researchers whose focus of inquiry pursues an interpretive interest this can invoke a somewhat different set of research acts. There is an acknowledgment that art practice is not only a personal pursuit but also a public process that can change the way we understand things. Consequently, the ideas expressed and communicated have an interpretive utility that assumes different textual forms as others make sense of what it is artists have to say through what it is they see. Interpretive research acts build on the rich conceptual traditions associated with image making whose purpose is to open up dialogue between the artist and viewer, and among an interpretive community whose interests may cut across disciplines. The linguistic turn of postmodernism has done much to disrupt the easy equation that presumes an artwork and its 'reading' by viewers is a simple matter of encoding and decoding visual forms. Interpretive acts open up the space among the artist, artwork, and the setting as different interests and perspectives are embraced. New understandings result as they are filtered through the interpretive community of art writers and theorists. As Arthur Danto (1981) reminds us You can call a painting anything that you choose, but you cannot interpret in any way you choose, not if the argument holds that the limits of knowledge are the limits of interpretation (Sullivan, 2006, pp. 31-32). 
Toda la reflexión anterior sobre la pedagogía, como acto de construcción de conocimiento que impacta decisivamente las dinámicas creativas del artista, debe ubicarse también aquí en la medida en que la pedagogía del arte es comunicación transformante y en que, vivida radicalmente, es investigación-creación.

Bell lo ha descrito de forma precisa: dentro de una práctica artística reflexiva se produce una forma específica de "saber comunicativo", generado desde un impulso pedagógico por compartir comprensiones críticas con otros. Se produce, por tanto, una complejidad emergente de conceptos, de refinamiento de técnicas artísticas y de unidad dentro de una comprensión estética compartida (característica de las artes creativas). Así, Bell concluye que:

The rational scrutiny of art practices and of creative arts pedagogy is just as likely to constitute an effective starting point for elaborating a model of research through creative practice than any attempt to apply a generic model of research that is inevitably unresponsive to the distinctiveness of the creative and performing arts as intellectual endeavors (Bell, 2006, pp. 86-87).

Sullivan también ha propuesto que un artista desarrolla actos críticos. Este autor diría que todo artista quiere, de alguna manera, "cambiar el mundo".

Art practice also draws on critical research acts and this stance has always been part of the history of art. There is an enactive or 'doing' element here, for critical action implies both a reactive and proactive stance, which is responsive to circumstances and contexts that require attention. Artworks have long been used as an instrument of social and political action, yet artists are sometimes hard pressed to show what their actions actually achieve. For some arts commentators, the presumed social role of artistic inquiry is misguided and should be left to the social scientists and political theorists (Hughes, 1993). Maxine Greene (2003), however, is fond of saying that art cannot change the world, but it can change someone who can. She talks of the "social imagination" as a site where what is possible can take flight, and where incompleteness and uncertainty are relished as habits of mind. For an arts researcher inspired by a call to critical action, any inquiry is undertaken for personal and public ends. A questioning attitude that is socially and culturally directed readily maps onto methods of inquiry and research acts that are responsive and exploratory. Yet the most crucial element within this inquiry process is the need to be able to create forms from which critical options can be more clearly assessed and addressed. This will require moving in and beyond the comfort of prescribed discipline knowledge, as issues and concerns demand approaches where new perspectives are opened up. Consequently it is the creation of new opportunities to see beyond what is known that has the potential to lead to the creation of new knowledge (Sullivan, 2006, p. 33).

Finalmente, está la contribución de esta investigación, y de este artículo: todo proceso creativo implica cierta medida de recolección, ordenamiento y sistematización de infor- 
mación. Los llamaremos como actos metodológicos. Desmond Bell, refiriéndose al campo de las artes audiovisuales, afirma que:

Research undertaken prior to a creative media project whether conducted in the academy or within a professional practice, is clearly part of the preparation for the intended production and as such is primarily shaped by professional considerations rather than by traditional disciplinary and academic ones. For example, television and film producers working in the factual field hire specialist picture researchers to locate relevant archival images. They call on the services of specialist academics such as historians to develop scripts and programme treatments. Similarly, the development of fiction scripts rests on extensive research into the cultural and historical location of events, the language, habit, psychological motives of characters and into the visual elements that make up the film's 'look'. However, such 'background research' is rarely of a primary nature and is harnessed to the demands of what we could call 'informed programme production' rather than disinterested knowledge generation within a discipline (Bell, 2006, pp. 85-86).

Desde estas cinco categorías (actos teoréticos, actos formativos, actos interpretativos, actos críticos y actos metodológicos), es posible abordar el análisis de prácticas creativas en el arte en general y, para lo que nos interesa, es posible entender y comprender las prácticas creativas en el cine de ficción. Esa es nuestra apuesta.

\section{Algunas conclusiones}

Haroldo de Campos, el gran poeta brasilero, releyendo a Sergei Einsenstein, Ernest Fenellosa y Ezra Pound, ha reiterado el carácter icónico de la escritura ideográfica china, una vieja tradición de los poetas. De esta forma, Ernest Fenellosa, traducido al portugués por Campos, creía que los ideogramas chinos cargaban sentido, no sólo de cosas sino sobre todo de acciones y movimientos. Cada ideograma, según Fenellosa, evocaba movimiento, tanto en los ideogramas individuales como en los compuestos. Así, en el proceso de composición, de “juntar” dos imágenes, no sólo se produce una tercera, sino que se sugiere una relación fundamental entre ambas. Fenellosa ofrece varios ejemplos:

- El ideograma para "comensal” muestra, según Fenellosa, un "hombre" y una "hoguera”.

- El ideograma para "primavera" muestra un "sol" y el "florecer de las plantas".

- El ideograma para "macho" muestra un "campo de arroz" y "lucha".

Sergei Eisenstein, también traducido al portugués por Campos, perseguía todos los posibles entrecejos de la noción de montaje cinematográfico. Para él, la escritura china y japonesa eran formas primigenias de montaje y, al "juntar" dos imágenes, se creaban nuevos sentidos que los componentes originales, por si mismos, apenas podían evocar. También proporcionaba varios ejemplos: 
-El ideograma para "ladrar", según Eisenstein, muestra un "perro" y una "boca".

-El ideograma para "gritar" muestra una "boca" y un "niño".

-El ideograma para "cantar" muestra una "boca” y un "pájaro".

-El ideograma para "tristeza" muestra un "cuchillo" y un "corazón”.

No se trata aquí de simplificar un debate lingüístico, crítico y literario muy complejo y, tal como lo rastreaba el mismo Campos, los tres intelectuales y poetas citados eran grandes creadores pero no necesariamente los mejores sinólogos o unos traductores expertos (de Campos, 2000).

Sin embargo, podríamos preguntarnos, como se hizo al inicio de este artículo: ¿qué pasa cuando la "investigación" se encuentra con la "creación"? ¿Qué ocurre cuando la imagen/palabra "investigación” se confronta con la imagen/palabra "creación"? Y usando la anterior reflexión, ¿qué ocurre cuando el ideograma "investigación" se amalgama con el ideograma "creación"? Nos precedió también la inquietud de si ¿el arte es investigación? Hemos tratado de responder a estas preguntas y, en el intento, hemos encontrado varias posibles acepciones:

- La creación basada en la investigación.

- La investigación sobre la creación.

- La creación como investigación.

- La investigación como creación.

- El performance como investigación.

- El arte en la investigación.

- La investigación-creación como forma de vida.

- La investigación en la creación.

- La investigación para la creación.

- La pedagogía como investigación-creación, e, incluso,

- La creación sin investigación.

Tal riqueza de vida, y tales minucias de sentido, es lo que produce la relación entre "investigación" y "creación”. Ante tal variedad, y tal movimiento, la investigación-creación en la América Latina requerirá, en el futuro, de un dinámico profundizar y ahondar en un debate y en una epistemología pero, sobre todo, requerirá ahondar en las posibilidades de una práctica. Y no sólo como respuesta a un medio institucional y político hostil o, al menos, indiferente, sino como necesidad de relanzar y perfilarse como un proyecto intelectual propio y creativo.

\section{Notas}

1. Afirma Graeme Sullivan que estas diferencias geográficas se explican por el hecho de que los conceptos de "arts-based research and arts-informed research in its various forms (are) being taken with enthusiasm within the United States and Canada”. 


\section{Referencias}

\section{Citas:}

ACOFARTES (2015), "Informe de la Presidencia. Mesa de trabajo de artes, arquitectura y diseño", Bogotá.

Andrews, E. (2009). "Arts-based Research: An Overview”, en http://www.personal.psu.edu/ eja149/blogs/elizandrews/2009/11/arts-based-research-an-overview.html. Recuperado el 14 de octubre de 2016.

Bamford, A. (2005). The Impact of the Arts in Education: A Global Perspective on Research. World Conference on Arts Education. Building Creative Capacities for the 21st Century, Lisboa.

Barriga Monroy, M. (2011). "La investigación-creación en los trabajos de pregrado y postgrado en educación artística”, en El Artista \#8, Bogotá, Universidad Distrital Francisco José de Caldas.

Bayazit, N. (2004). "Investigating Design: A Review of Forty Years of Design Research". Design Issues 20.1: 16-29.

Bell, D. (2006). "Creative Film and Media Practice as Research: In Pursuit of That Obscure Object of Knowledge". Journal of Media Practice Vol. 7, Intellect Ltd: 85-100.

Borgdorff, H. (2006). El debate sobre la investigación en las artes. Amsterdam: Amsterdam School of the Arts.

De Campos, H. (Ed.) (2000). Ideograma. Lógica, poesia, linguagem. São Paulo: Editora da Universidade de São Paulo.

Candy, L. (2006). "Practice-based Research: a Guide”, en CCR Report Vol. 1.0.: 1.

Casas, M. V. (2013). "Una aproximación al estado del arte en Colombia y otros países sobre la discusión investigación-creación en artes avances y propuestas", en Casas MV (Comp.), Memorias del evento Valoración de los procesos de creación artística y cultural en el marco de la acreditación de programas. Bogotá: Colciencias-Consejo Nacional de Acreditación - CNA.

Daza Cuartas, S. (2009). “Investigación-Creación. Un Acercamiento a la Investigación en las Artes", en Horizonte Pedagógico. 11.1: 87-92.

FEMIS. Doctorat SACRe (2016), en http://www.femis.fr/doctorat-sacre. Recuperado el 4 de diciembre de 2016.

International Federation of Theatre Research (2016). “Performance as Research”, en https:// www.iftr.org/working-groups/performance-as-research. Recuperado el 14 de octubre de 2016.

Irwing, R. (2016). “A/r/tography”, en: http://artography.edcp.educ.ubc.ca/. Recuperado el 14 de octubre de 2016.

Knowles, G. \& Ardra L. Cole (Editores) (2014). Handbook of the Arts in Qualitative Research: Perspectives, Methodologies, Examples, and Issues. London: Kindle Edition.

Le Figaro (2016). “SACRe, un doctorat inédit alliant sciences et arts”, en http://www.lefigaro. fr/formation/2012/10/16/09006-20121016ARTFIG00573-sacre-un-doctorat-ineditalliant-sciences-et-arts.php. Recuperado el 4 de diciembre de 2016.

Rust, Ch., Mottram \& Jeremy Till (2007). "Practice-Led Research in Art, Design and Architecture" en AHRC Research Review, November. 
Sullivan, G. (2006). "Research Acts in Art Practice" en Studies in Art Education 48.1: 19-35. University of Bristol (2016). "Practice as Research in Performance: 2001-2006”, en http:// www.bris.ac.uk/parip/ Recuperado el 14 de octubre de 2016.

Wikipedia (2016). “Master of Fine Arts”, en https://en.wikipedia.org/wiki/Master_of_Fine_ Arts. Recuperado el 16 de noviembre de 2016. . (2016). “Practice research”, en https://en.wikipedia.org/wiki/Practice_research. Recuperado el 14 de octubre de 2016. . (2016). "Research_Assessment_Exercise", en https://en.wikipedia.org/wiki/ Research_Assessment_Exercise. Recuperado el 4 de diciembre de 2016.

\section{Libros:}

Barone, T. \& Elliot W. Eisner (2008). Arts Based Research. London: Sage Publications.

Cahnmann-Taylor, M. \& Richard Siegesmund (Compiladores) (2008). Arts-Based Research in Education: Foundations for Practice. New York: Inquiry and Pedagogy. Across Diverse Contexts Series. Routledge.

Fuschini, L., Simon Jones \& Baz Kershaw (2009). Practice-as-Research: In Performance and Screen,. London: Palgrave-Macmillan.

Haywood Jr. \& James Rolling (2013). Arts-Based Research Primer. Nueva York: Peter Lang. Irwing, R. \& Alex de Cosson (Eds.) (2004). A/r/tography: Rendering Self Through Arts-Based Living Inquiry. Vancouver: Pacific Educational Press.

Knoles, J., Gary Ardra \& L. Cole (2008). Handbook of the Arts in Qualitative Research: Perspectives, Methodologies, Examples, and Issues. London: Sage Publications.

Leavy, P. (2015). Method Meets Art, Second Edition: Arts-Based Research Practice. New York: Guilford Press.

Mcniff, S. (2009). Art as Research: Opportunities and Challenges. London: Intellect Ltd.

Riley, S. \& Lynette Hunter (Comp.) (2009). Mapping Landscapes for Performance as Research: Scholarly Acts and Creative Cartographies. London: Palgrave-MacMillan.

Springgay, S., Rita L. Irwin, Carl Leggo, \& Peter Gouzouasis. (Comp.), (2008). Being with A/r/tography. Roterdam: Sense Publishers.

Sullivan, G. (2005). Art Practice as Research: Inquiry in the Visual Arts. London: Sage Publications.

\section{Bibliografía adicional no citada}

. (2004). "Research in the Creative and Performing Arts 'the RAE and Research in the Creative \& Performing Arts. Review of Research Assessment'-- an AHRB Paper [1]". Journal of Visual Art Practice 3.1: 75-78.

Amerika, M. (2004). “Anticipating the Present: An Artist's Intuition” en New Media \& Society 6.1: 71-78.

Baker, C., Max Schleser, and Kasia Molga (2009). “Aesthetics of Mobile Media Art”. Intellect Ltd., 101-22. Vol. 10.

Berthe C., K. (2007). "Why Give a Ph. D. In Creative Art?” Parnassus 13.2.

Candy, L. (2007). "Constraints and Creativity in the Digital Arts”. Leonardo 40.4: 366-67. . (2002). "Introduction: Creativity and Cognition. Part I: Perspectives from the Third Symposium”. Leonardo 35.1: 55-57. 
. (2002). "Introduction: Creativity and Cognition: Perspectives from the Third Symposium: Part 2”. Leonardo 35.3: 313-14.

Candy, L. and Ernest A. Edmonds (2011). Interacting: Art, Research and the Creative Practitioner. Faringdon, Oxfordshire: Libri Pub.

Candy, L. et al. (1999). Creativity \& Cognition. Proceedings of the Third Creativity \& Cognition Conference, Loughborough University, Loughborough, Uk, October 10-13, 1999. Web $<$ http://www.columbia.edu/cgi-bin/cul/resolve?clio10112893>.

. (1993). "Creativity \& Cognition: Proceedings of the ... Creativity \& Cognition Conference”. New York: NY: Association for Computing Machinery.

Candy, L., Sam Ferguson, and SpringerLink (Online service) (2014). Interactive Experience in the Digital Age Evaluating New Art Practice. Web <http://www.columbia.edu/cgi-bin/ cul/resolve?clio10805290>.

Cross, N. (1999). "Design Research: A Disciplined Conversation” Design Issues 15.2: 5-10.

Crouch, C. (2007). "Praxis and the Reflexive Creative Practitioner." Journal of Visual Art Practice 6.3: 105-14.

Dowmun, T., and Rosie Thomas (2005). "Supervising and Examining Practice-Based Phds in the Moving Image”. Intellect Ltd., 121-26. Vol. 6.

Edmonds, E. and Linda Candy (2010). "Relating Theory, Practice and Evaluation in Practitioner Research”. Leonardo 43.5: 470-23.

Fanning, Ralph, Stephen C. (1940). Pepper, and Millard Sheets. "Why Give a Ph. D. In Creative Art?" Parnassus 12.8: 2-3.

Gablick, S. (1984). ¿Ha muerto el arte moderno? Nueva York: Thames y Hudson.

Garfield, R. (2007). "Articulating a Position through Research: The Practice-Led Phd, a Case Study". Journal of Media Practice 8.2: 221-34.

. (2007). "Articulating a Position through Research: The Practice-Led Phd, a Case Study”. Intellect Ltd. Intellect Ltd., 221-34. Vol. 8.

Glaveanu, V. (2013). "Creativity and Folk Art: A Study of Creative Action in Traditional Craft”. Psychology of Aesthetics, Creativity \& the Arts 7.2: 140-54.

Gomez Muntane, M. (2006). “Campos, temas y metodologías de la investigación relacionada con las artes: algunas reflexiones sobre el caso de la musicología, en M. Gómez Muntané, F. Hernández Hernández y H. J. Pérez López, Bases para un debate sobre investigación artística, Barcelona, Ministerio de Educación y Ciencia.

Grierson, E. (2014). "Scrutinizing Studio Art and Its Study: Historical Relations and Contemporary Conditions”, en Journal of Aesthetic Education 44.2: 111-23.

Kerrigan, S. (2013). "Accommodating Creative Documentary Practice within a Revised Systems Model of Creativity" en Journal of Media Practice 14.2: 111-27.

Khan, M. (2013). "Alternative Paradigm of Research in the Creative Arts and Design", en Putaj Humanities \& Social Sciences 20: 357-69.

Knubsen, E. (2007). "Heart of Gold: Fact and Mysticism in Documentary Form a Research Report” en Intellect Ltd. Intellect Ltd:. 49-61. Vol. 8.

Vol. 3 . . (2002). “Creation and I, Me and My Work”. Intellect Ltd. Intellect Ltd., 107. . (2002). "Doctorate by Media Practice." Intellect Ltd. Intellect Ltd. 179. Vol. 3. Print. 
Leggett, M. (2006). "Interdisciplinary Collaboration and Practice--Based Research". En Convergence: The Journal of Research into New Media Technologies 12.3: 263-69.

Lord, A. (2012). "Qualitative Research in the Creative Arts" en Creative Approaches to Research 5.1: 58-76.

Mclean, C. (2011). Creative Arts in Research for Community and Cultural Change. Calgary: Detselig Enterprises.

Nash, M. (2013). “Unknown Spaces and Uncertainty in Film Development" en Journal of Screenwriting 4.2: 149-62.

Nelmes, J. (2007). "Some Thoughts on Analysing the Screenplay, the Process of Screenplay Writing and the Balance between Craft and Creativity". Intellect Ltd. Intellect Ltd.: 10713. Vol. 8.

Redvall, E. (2012). "A Systems View of Film-Making as a Creative Practice". Northern Lights: Film \& Media Studies Yearbook 10.1: 57-73.

Troshani, F. (2013). "Interacting: Art, Research and the Creative Practitioner". Leonardo 46.5: 506-07. Print.

\begin{abstract}
The article seeks to outline the different meanings of the relationship "research/ art", in order to take advantage of its heuristic capacity. The article emphasizes the concept "research/art" in the audiovisual arts, placing the notion in the wider debate of the arts, of artistic creation and, indirectly, in the fields of design and architecture. It is said that the "monolithic" use of the neologism "research/art" in the Spanish contrasts with the variety of meanings that characterize the concept in English. Several concepts are defined: "practice-based research", "practice-led research", "practice as research", "practice as research in performance", "arts-based research", "arts-informed research", "a/r/tography", and "research acts in art practice". A history of the debate on the subject, and the fundaments of a state of the art, is proposed, guided by the question: is art research? Finally, the article emphasizes on "research acts in art practice" in concrete and historical processes of artistic creation and, based on the categories of Australian artist and academician Graeme Sullivan, five categories are proposed to understand the research acts in which concrete artists and filmmakers are involved.
\end{abstract}

Key words: Research - artistic creation - creativity - creative processes - film - art practice - art - research acts in art practice.

Resumo: Este artigo procura delinear os diferentes significados do conceito de "pesquisa-criação" para aproveitar sua capacidade heurística. Alega-se que surpresas o uso monolítico do neologismo em espanhol, em contraste com a variedade de nuances de significância que caracterizam o conceito em inglês. Conceitos são definidos como "pesquisa com base na criação", "pesquisa voltada para a criação", "criação como pesquisa", "performance como pesquisa", "o uso das artes na pesquisa", "arte", "criação como pesquisa". Uma história do debate sobre o tema e lançar as bases de um estado de arte guiado pela questão: é arte, pesquisa? Finalmente, a ênfase é colocada na criação como pesquisa em processos 
concretos e criação artística histórica e, a partir das categorias propostas pelo artista e pesquisador australiano Graeme Sullivan,cinco categorias são formuladas para compreender os atos investigativos em que artistas e cineastas específicos estão envolvidos.

Palavras chave: Pesquisa - pesquisa-criação - criação artística - criatividade - processos criativos - cinema - práticas artísticas - arte - atos investigativos na arte.

[Las traducciones de los abstracts fueron supervisadas por el autor de cada artículo] 